\title{
Efecto pantalla y efecto Sísifo: dos fenómenos didácticos en la resolución de problemas por transferencia analógica
}

\section{Screen effect and Sisyphus effect: two didactic phenomena in problem-solving by analogical transfer}

\section{Carlos B. Gómez ${ }^{1}$ \\ Joan Josep Solaz-Portolés ${ }^{2}$ \\ Vicent Sanjosé ${ }^{2}$}

(1) Florida Universitaria, Catarroja, España

(2) Universitat de València, España

Resumen: Se presenta un estudio cualitativo, basado en entrevistas semi-estructuradas, de 2 casos que muestran dos fenómenos didácticos de interés en la resolución de problemas por transferencia analógica. El primero muestra un 'efecto pantalla', en el cual el sujeto no puede acceder a la estructura de los problemas a partir de los enunciados. En el segundo caso, el sujeto no logra alcanzar un estado estable de comprensión, a pesar de su avance, y por tanto acaba por retroceder a un estado de ausencia de conflicto cognitivo, pero indeseado desde el punto de vista didáctico ('efecto Sísifo'). Se discuten las posibles implicaciones didácticas de estos casos.

Palabras clave: Resolución de problemas; Transferencia analógica; Superficie y Estructura de problemas; Entrevista semiestructurada; Educación secundaria.

Abstract: A qualitative study, based on semi-structured interviews to two students, is presented. These two cases represent interesting learning phenomena related to problemsolving by analogical transfer. The first one shows a 'Screen effect' in which the student cannot access to the problems structure from their statements. The second case shows the 'Sisifo effect': the subject is progressing up on the 'learning slope', but is not able to arrive to a steady, new cognitive state. As a consequence, he/she returns to a 'lower' cognitive state, free from cognitive conflict but undesirable from the educational point of view. The possible educational consequences of these cases are discussed.

Key Words: Problem solving; Analogical transfer; Problems surface and structure; semistructured interviews; Secondary education.

(Fecha de recepción: julio, 2013, y de aceptación: septiembre, 2014)

DOI: 10.7203/DCES.28.2633 


\section{Introducción}

Cuando se instruye en resolución de problemas en el aula, una estrategia frecuentemente utilizada es la transferencia analógica (Gómez-Ferragud, Solaz-Portolés y Sanjosé, 2012; Bernardo, 2001; Reed, Dempster y Ettinger, 1985; Gick y Holyoak, 1983): el profesor resuelve un conjunto de problemas que tienen algo en común, alguna regla, procedimiento, principio, ley o teorema $\mathrm{y}$, a continuación, plantea problemas análogos a los estudiantes.

Chen y Klahr (2008) diferencian cuatro etapas el transfer analógico: a) Codificación del problema propuesto; b) Acceso a un problema análogo-fuente; c) Mapping, o correspondencia, entre el problema propuesto y el análogofuente; d) Ejecución de estrategias para solucionar el problema propuesto. La codificación permite la 'indexación' del problema en el 'archivo' de la memoria a largo plazo (MLP), de modo que pueda luego ser re-activado cuando el sujeto resolutor busque un problema análogo ('análogo fuente') que le ayude a resolver el problema que se le ha planteado ('diana'). Una vez reactivado el análogo fuente, el resolutor debe establecer una correspondencia o mapping entre las variables características de ambos problemas y así, el análogo-fuente queda listo para ser usado en la resolución del diana.

Por tanto, la transferencia analógica requiere que el alumno establezca una analogía entre una situación problemática conocida y una nueva (Gent- ner, 1983). Cuando los problemas tienen enunciado, es usual que la analogía entre problemas diferentes se tenga que construir a partir de la identificación y construcción de relaciones (mapping) entre elementos presentes en ellos, característicos de los problemas. Holyoak (1984) ha diferenciado dos grupos de características definitorias de los problemas matemáticos: la 'Superficie' y la 'Estructura'. La Superficie describe la situación problemática en el mundo real, y alude a objetos y eventos en términos concretos (no abstractos). En la educación Secundaria, un grupo importante de problemas académicos tienen naturaleza algebraica. La Estructura de un problema algebraico está determinada básicamente por "cómo se relacionan las cantidades unas con otras más que por cuáles son esas cantidades" (Novick, 1988; p. 511). En los problemas que vamos a considerar en este estudio, las ecuaciones resumen las relaciones entre cantidades. Así pues, desde el punto de vista teórico la relación entre dos problemas puede caracterizarse en términos similitud superficial y/o estructural (Holyoak y Koh, 1987).

Los problemas utilizados en este trabajo se pueden relacionar mediante un esquema abstracto común. Este esquema implica dos ecuaciones lineales con una solución única, esto es, se representan geométricamente por dos líneas rectas que se cortan:

Ecuación 1: $y_{1}=y_{10}+r_{1} x$

Ecuación 2: $y_{2}=y_{20}+r_{2} x$ 
donde $y_{10}$ e $y_{20}$ son los puntos de corte con el eje de ordenadas y $r_{1}, r_{2}$ son las pendientes de las rectas (o 'razones' de crecimiento o decrecimiento de la variable dependiente). Denominaremos 'Alcanzar' la estructura correspondiente a pendientes positivas y de diferente valor, y ordenadas en el origen también diferentes. Si las pendientes de las rectas tienen signo y valor diferentes, $y$ sus ordenadas en el origen son también distintas, la estructura resultante será denominada 'Encontrar'1.

En investigaciones anteriores (Reed, 1987; Gómez-Ferragud, Solaz-Portolés y Sanjosé, 2013a, Gómez-Ferragud, Solaz-Portolés y Sanjosé, 2013b), este planteamiento teórico ha servido de base para estudiar las dificultades que los estudiantes de Secundaria tienen en las fases a, b y c del transfer según Chen y Klahr (op.cit.). Se ha analizado si los estudiantes prestan más atención a los elementos superficiales o a los estructurales a la hora de construir esas analogías. Entre los resultados de interés se encontró que muchos estudiantes focalizan su atención sobre la pregunta o demanda del problema: el nombre de la magnitud implicada, su rol algebraico o aritmético, su posición dentro del enunciado, etc. (Castro, Rico, Batanero y Castro, 1991). Muchos estudiantes usan una estrategia "hacia atrás", desde la pregunta del problema hacia los datos, para intentar resolverlo y, por tanto, los rasgos de la pregunta son elementos de primera importancia para ellos. También se encontró que las similitudes superficiales entre problemas parecían dificultar a los alumnos detectar sus diferencias estructurales. Con mucha frecuencia, los elementos superficiales fueron tomados como base de la codificación y categorización de los problemas (Gómez-Ferragud, SolazPortolés y Sanjosé, 2013c).

Los estudios estadísticos mencionados apuntan la existencia de algunos fenómenos didácticos interesantes, pero los procesos mentales implicados no han sido aún analizados. El objetivo del presente trabajo es indagar en las dificultades de los estudiantes durante el proceso de realizar una transferencia analógica entre problemas. Usaremos dos tareas, una de categorización de problemas, y otra de establecimiento de analogías entre problemas con diferentes relaciones analógicas entre ellos. En la segunda tarea usaremos entrevistas y protocolos think aloud para tratar de obtener evidencias de los procesos cognitivos y metacognitivos implicados en el establecimiento de analogías o diferencias entre problemas propuestos la segunda tarea. Atenderemos en especial a la influencia de elementos superficiales o estructurales de los problemas. Finalmente, intentaremos extraer las posibles consecuencias didácticas y aprender de estos análisis.

\footnotetext{
${ }^{1}$ Los nombres de ambas estructuras se han tomado de la cinemática de móviles: un vehículo que alcanza a otro moviéndose por la misma carretera (Alcanzar), o dos vehículos que se mueven en sentido opuesto en la misma carretera y acaban encontrándose (Encontrar).
} 
En este trabajo mostramos el análisis de dos entrevistas que ejemplifican dos fenómenos didácticos de interés: el 'efecto pantalla' y el 'efecto Sísifo'. En el primer caso, los elementos superficiales de los problemas impiden al estudiante acceder a la estructura de los mismos y establecer analogías en función de ella. Sin embargo, cuando el estudiante es situado en la representación abstracta, matemática de los problemas, no tiene ninguna dificultad en comprenderla y vincular las ecuaciones con las ideas apropiadas de los enunciados. El segundo caso alude a Sísifo, rey de Corinto mencionado en La Odisea, que fue condenado por los dioses a pasar toda la eternidad en el infierno empujando hacia arriba una pesada roca por la ladera de una montaña. Antes de alcanzar la cima, la roca caía a la base y Sísifo tenía que repetir el esfuerzo. En este caso, el sujeto analizado avanza con esfuerzo a lo largo de la entrevista por un camino correcto, pero no alcanza el estado final de equilibro cognitivo asociado con una comprensión completa. Entonces, el sujeto acaba regresando a un estado que, para él, resuelve su conflictivo cognitivo, pero que no es aceptable desde el punto de vista instruccional, con lo cual el estudiante tendrá que repetir el esfuerzo más adelante.

\section{Método}

\section{Estudiantes participantes}

En la primera tarea participaron 21 sujetos pertenecientes a un grupo intacto de 3er curso de $\mathrm{ESO}$ ( $9^{\circ}$ grado) de un centro público de enseñanza en un barrio de nivel socio-económico medio en una ciudad de unos 80.000 habitantes. Aunque los sujetos no presentaron a priori ninguna característica diferenciadora del resto de la población de ese curso, se trató de una muestra de conveniencia. En la segunda tarea, se excluyeron aquellos estudiantes que mostraron poco interés o entusiasmo en la primera prueba. Se entrevistaron 11 estudiantes que representaron diferentes criterios en la tarea 1: 4 estudiantes representaron a los que usaron un criterio experto correcto; 4 estudiantes basaron su agrupación en el nombre de la magnitud incógnita; 1 estudiante utilizó un criterio mixto cruzando la Estructura con el Contexto de los problemas; 2 estudiantes usaron criterios incoherentes. De estos estudiantes, 2 mostraron dos fenómenos didácticos de interés que se muestran en este trabajo. Uno/a de ellos basó la tarea 1 en un criterio superficial asociado al nombre de la magnitud incógnita. El/la otro/a es el estudiante que utilizó el criterio mixto basado en la conjunción Estructura $\mathrm{x}$ Contexto.

\section{Diseño y materiales}

El estudio implicó dos tareas. Primero se realizó una categorización de problemas (Chi, Feltovich y Glaser, 1981) para activar los procesos básicos implicados en la resolución de problemas por transferencia analógica: codificación, indexación y relación entre problemas. La tarea propuesta al grupo completo de participantes consistió en agrupar enunciados 
de 8 problemas (ver Anexo 1) en conjuntos, dentro de los cuales sus elementos se resolvieran con las mismas ecuaciones.

La segunda parte del estudio se dedicó al análisis detallado de los procesos mentales de los estudiantes cuando intentan resolver un problema mediante transferencia analógica. La tarea planteada consistió en relacionar un problema propuesto ('diana') con diferentes problemas 'fuente', y explicar cuál o cuáles de los 'fuentes' resultaban de más ayuda para resolver el 'diana'. Esta tarea se realizó individualmente, mediante una entrevista extensa semiestructurada a cada participante con instrucciones para narrar en voz alta sus pensamientos e intenciones. Se buscó asociar procesos mentales con el establecimiento de analogías de distinto tipo (Clement, 1988) y diferencias entre problemas. La entrevista consistió en las siguientes fases: 1) Recordatorio y clarificación del criterio empleado en la tarea de agrupación; 2) Suministro del problema diana y diferentes problemas fuente para el establecimiento de analogías (y diferencias) que sirvan para la resolución del diana por transferencia; 3) Explicitación de las ecuaciones que resuelven correctamente los problemas fuente y revisión de las analogías y diferencias establecidas. Los problemas utilizados en la entrevista se encuentran en el Anexo 2.

\section{Análisis}

Todos los problemas utilizados en nuestro estudio están caracterizados por 3 variables o factores: 1.-La Superficie, cuyos elementos son irrelevantes para resolver los problemas; 2.-La Estructura, o relaciones entre las cantidades dadas y la cantidad demandada (la incógnita); 3.-La variable demandada, o Incógnita, que se puede diferenciar por la magnitud que la define, y por el rol algebraico que juega en las ecuaciones (como 'x' o como 'y'). La magnitud demandada está asociada con la temática científica del problema. Se ha probado que los estudiantes de menor conocimiento previo focalizan su atención y son muy influidos por el nombre de la incógnita, su posición en un enunciado o su rol matemático, etc. (GómezFerragud et al, 2013c).

En la Tabla I se analiza uno de los problemas utilizados, para mostrar sus elementos superficiales, aquellos de relevancia estructural y la incógnita.

Los problemas fuente usados durante la entrevista individual se relacionaron con el problema diana de formas muy bien definidas (Anexo 2). Los 3 problemas 'fuente lejanos' que se suministraron en la fase 2A de la entrevista, sólo comparten uno de sus elementos característicos con el 'diana' (problemas 'fuente lejanos'). Así, el fuente 'Similar lejano' compartió con el Diana los objetos y situación, pero no la estructura, ni la temática científica (magnitudes) que afecta el nombre de la variable incógnita. El fuente 'Isomorfo lejano', compartió la estructura con el Diana, pero no el resto de características (temática, incógnita, objetos y situación). Asimismo, el fuente 'Relacionado lejano' compartió con el Diana el nombre 


\section{Tabla I. Componentes superficiales e ideas de relevancia estructural del enunciado de uno de los problemas utilizados en el estudio}

\section{Elementos Superficiales}

\section{Objetos y entidades:}

Globos; Bombas Neumáticas; gas; depósito.

\section{Eventos:}

Considerar dos globos; Conectar a la vez dos bombas neumáticas idénticas; Extraer gas; Inyectar gas; Disminuir volumen; Aumentar volumen; Transferir gas.

Magnitudes implicadas: $\mathrm{cm}^{3} ; \mathrm{g} ; \mathrm{cm}^{3} / \mathrm{g}$

Magnitud-incógnita: gramos

\section{Ideas con relevancia estructural}

\section{Traducción algebraica de las ideas} con relevancia estructural

1. El volumen del globo A va disminuyendo a razón de $20 \mathrm{~cm}^{3} / \mathrm{g}$

2. El volumen del globo $B$ va aumentando a razón de $30 \mathrm{~cm}^{3} / \mathrm{g}$ :

3. Antes de conectar las bombas, el globo $\mathrm{A}$ tiene un volumen de $2000 \mathrm{~cm}^{3}$

4. Antes de conectar las bombas el globo B está vacío

$\Delta V_{A}=-20 \Delta m_{A}$

$\Delta V_{B}=+30 \Delta m_{B}$

$V_{A}=2000-20 \Delta m_{A}$; en cualquier momento a partir de la conexión de la bomba $\mathrm{A}$. $V_{B}=+30 \Delta m_{B} ;$ en cualquier momento a partir de la conexión de la bomba $B$

\section{Pregunta}

¿Cuántos gramos de gas se habrán transferido de A a B, cuando sus volúmenes sean iguales?
Traducción algebraica de la pregunta

encuentra $\Delta m_{B}$ cuando $V_{A}=V_{B}$ de la magnitud incógnita demandada, pero no la estructura, ni los objetos o la situación. Por su parte, los 2 problemas 'fuente cercanos' (fase 2B de la entrevista), sólo se diferenciaron del 'diana' en una característica constitutiva. El problema 'Isomorfo cercano', compartió con el Diana la estructura y el nombre de la magnitud incógnita, pero no los objetos ni la situación; el fuente 'Relacionado cercano' compartió con el Diana los objetos y situación, así como el nombre de la magnitud incógnita, pero no la estructura $^{2}$. Naturalmente, solamente los problemas Isomorfos (lejano y cercano) se resuelven exactamente con las mismas ecuaciones que el problema Diana.

${ }^{2}$ La estructura de este problema 'Relacionado cercano' corresponde 'mezclas', como el 'Relacionado lejano, REL1'. Por eso no lo denominamos 'Similar cercano', a pesar de que ahora comparte objetos y magnitudes con el Diana. El 'Similar lejano SIM1' de la parte anterior, tiene estructura 'Alcanzar'. 
Codificación del comportamiento de los sujetos durante la tarea de transferencia

Se utilizó la codificación propuesta por Codina, Castro y Cañadas (2011, pp. 160-161) para el análisis de los protocolos de resolución de problemas, aunque adaptada a la tarea de transfer analógico. Esta codificación se apoya, a su vez, en el trabajo de Artzt y Armour-Thomas (1992), y en la propuesta anterior de Schoenfeld (1985). Tanto en la etapa en la que se suministró el problema a resolver ('Diana') como en la que se ofrecieron los problemas 'fuente', se diferenciaron los episodios: Lectura, Análisis y Exploración. La ejecución de la resolución, propiamente dicha, no fue abordada en este trabajo: la tarea demandada a los sujetos fue la de establecer analogías y diferencias entre problemas, es decir, basarse en los problemas fuente para determinar cuál de ellos podría ayudar más a resolver el Diana, pero no ejecutar la resolución completa de éste.

Dentro del episodio de Exploración, se contempló específicamente el establecimiento de similitudes y diferencias entre problemas. Para esta parte tomamos como referencia el trabajo de Clement (1988) sobre el establecimiento y utilización de analogías para resolver problemas de física de cierta dificultad. Dentro de esta fase, diferenciamos las etapas y episodios incluidos en la Tabla II.

\section{Procedimiento}

En una sesión ordinaria de clase, se llevó a cabo la tarea de agrupación de problemas (30 min). Se repartió y leyó la hoja de instrucciones, se realizó una tarea de práctica y a continuación los estudiantes categorizaron y agruparon los 8 problemas propuestos, a partir de la lectura de sus enunciados (sin resolverlos efectivamente).

El estudio de transfer analógico, realizado mediante entrevistas, se inició una semana más tarde y se prolongó 2 meses. Las entrevistas se realizaron en un espacio distinto al aula, en condiciones de privacidad y confort suficiente del estudiante. Se obtuvieron los permisos necesarios de profesores y padres para entrevistar y grabar las sesiones en video. La cámara se dispuso de tal forma que el rostro del participante nunca estuvo en plano, pero sí los problemas y las acciones del sujeto. Cada problema se mostró sobre una cartulina de diferente color, de modo que fue siempre perfectamente distinguible en las imágenes grabadas. Las acciones y verbalizaciones de cada estudiante pudieron ser asociadas claramente con los distintos problemas.

Uno de los investigadores (CG) actuó como entrevistador. La entrevista siguió un protocolo planificado que se ajustó a cada sujeto. Comenzó recordando la agrupación realizada por el sujeto en la primera tarea. El propósito fue activar los criterios de codificación y categorización de problemas, que son procesos subyacentes en el establecimiento de analogías entre problemas. Luego, el entrevistador planteó a cada sujeto la segunda tarea: determinar cuál o cuáles de los problemas 'fuente' suministra- 
Tabla II. Etapas, episodios y elementos contemplados en la codificación de analogías entre los problemas fuente y el problema diana. Los códigos se expresan entre corchetes

\begin{abstract}
A. Analogías y diferencias entre el(los) problema(s) 'Fuente(s)' y el 'Diana'.
Episodio 1 [GA/GD]: GENERACIÓN de una ANALOGÍA/DIFERENCIA entre problemas. Distinguimos 3 tipos de analogías o diferencias (Clement, 1988): a) aquellas basadas en un (o más) principio(s) más general(es), del/de los cual(es) ambos problemas comparados son casos particulares o instanciaciones; b) identificar las ideas de ambos problemas que son equivalentes y no lo son, es decir, aquellas que podrían/no podrían intercambiarse entre ellos sin que cambiara su resolución (analogía/diferencia basada en una transformación), sin hacer referencia a una estructura abstracta más general; c) relacionar una idea o un rasgo del problema diana con otra idea o rasgo del problema fuente que desempeña/no desempeña el mismo rol, sin identificar correctamente las diferencias entre otras ideas (analogía basada en una asociación).
\end{abstract}

Episodio 2 [DA/DD]: DISCUSIÓN de la ANALOGÍA/DIFERENCIA establecida, revisión de la confianza en la analogía. Se atendió a las manifestaciones del sujeto al analizar la corrección de la analogía establecida.

Episodio 3 [UA/UD]: UTILIDAD de la ANALOGÍA/DIFERENCIA. Se atendió a las manifestaciones del sujeto sobre la utilidad de la analogía establecida para comprender y resolver el problema diana.

\title{
B. Comparación o relación entre problemas Fuente.
}

Idem etapa A, pero entre los problemas fuente entre sí.

dos era de mayor ayuda para resolver el problema 'diana', y explicar la razón. También recordó que el propósito del estudio era estudiar los procesos mentales que siguen los estudiantes cuando han de resolver problemas, y no juzgar la calidad de la resolución. Las entrevistas se articularon sobre un protocolo predefinido, aunque se adaptaron a cada participante. El entrevistador intervino siempre que se hizo necesario estimular al estudiante para que expresara en voz alta sus pensamientos, o clarificara sus acciones y sus objetivos. Se advirtió que las interrupciones en ningún caso se deberían a la correcta o incorrecta actuación del estudiante durante la ejecución de la prueba. La duración de las entrevistas fue variable, entre 15 y $25 \mathrm{~min}$.

\section{Estudio de casos}

A continuación se presentan porciones extensas de las entrevistas a dos 
estudiantes. Se ha respetado el progreso mental de los alumnos, de modo que los fragmentos eliminados son mínimos, y han sido sustituidos por narraciones (en cursiva).

En negrita se recogen las intervenciones literales del sujeto (S) y del entrevistador (E). En cursiva y entre corchetes se realizan los comentarios y análisis pertinentes. En la columna de la izquierda, se muestra el momento de cada segmento de la entrevista (minuto:segundo), y el código asociado con los 3 episodios definidos en el establecimiento de analogías y diferencias entre problemas, recogido en la Tabla II (GA/GD; DA/DD; UA/UD).

\section{Cuadro I. Caso MLR: Análisis del protocolo de la entrevista mostrando el 'efecto pantalla'}

Caso MLR: 'Efecto Pantalla', o imposibilidad de apreciar las diferencias estructurales entre problemas, a causa de sus similitudes superficiales.

\section{Min y Código Caso: MLR}

00:00-07:45 Fase 1 (resumen): En la tarea de agrupación, el sujeto definió los conjuntos de problemas según el nombre de la magnitud-incógnita. La fase 1 de la entrevista sólo sirve para confirmar ese criterio.

Fase 2: analogías y diferencias entre enunciados de problemas

08:10-09:02 El entrevistador explica la nueva tarea y su propósito, da las instrucciones y clarifica que sus intervenciones estarán destinadas a explicitar pensamientos del sujeto y no a juzgar la corrección o incorrección de los mismos. Primero entrega el problema Diana que el sujeto lee en silencio sin mostrar dudas ni pedir aclaraciones. A continuación el entrevistador entrega los enunciados de 3 problemas fuente (sin resolver). Los ubica en la mesa, frente al sujeto, en posiciones equidistantes a él. El sujeto tiene ante sí el Diana, y los 3 fuentes algo separados, pero al alcance de su vista. El entrevistador plantea la tarea con claridad y recuerda el procedimiento a seguir.

09:03 E: Entonces, mirando los tres problemas de las fichas [El entrevistador señala las 3 fichas de colores que contienen los problemas fuente], ¿cuáles crees que te ayudaran más o no te ayudarán para resolver el problema objetivo? Recuerda que puedes coger los problemas en el orden que quieras. Como están en cartulinas de colores la cámara recogerá qué problema estás mirando y puedes leer y releer cualquiera de ellos en todo momento...Recuerda también que te haré preguntas en función de lo que vayas haciendo... [nueva clarificación de las instrucciones y del procedimiento ]. Puedes empezar.

09:20-11:15 S: [El sujeto realiza primero un chequeo, una lectura general rápida de los 3 problemas fuente, comparando con el problema diana, sin tomar ninguno para procesarlo con calma. Emplea solamente 12 s para leer 3 problemas nuevos. Ello hace sospechar que el sujeto intenta 'buscar y detectar' la presencia de algún elemento predeterminado. Luego toma el problema SIM1 y lo sitúa ante sí. Lo lee en silencio y lo compara con el diana. A continuación toma el problema ISO1 y lo lee en silencio. Finalmente, lee en silencio el problema fuente restante, con igual pregunta al diana, el REL1. Lo hace desde lejos, sin modificar su posición].

11:16 S: [De pronto, con un gesto rápido, el sujeto toma el problema REL1 y lo coloca ante sí]. 
11:18 GD S: Es que en estos dos problemas [señala el SIM1 y el ISO1] las unidades que me dan son diferentes a las del problema que he de resolver... [Explicita diferencias, basadas en una característica superficial, entre SIM1 y DIANA, y entre ISO1 y DIANA. Es el primer rasgo en el que centra su atención. Nótese que es el mismo rasgo en que este sujeto basó su categorización de problemas en la primera tarea, que fue recordada en la fase 1 como introducción a ésta de transfer].

11:21-11:51 S: Voy a ver el otro [se refiere al problema REL1. El sujeto toma el problema rojo REL1 y lo sitúa delante de sí junto al problema ISO1. Luego compara ambos problemas con el diana de sendos vistazos. Retira de delante de sí el problema con igual estructura al fuente, ISO1, y luego se detiene a releer REL1].

11:52 GA S: Éste [señala el REL1] es el que veo más parecido. [El sujeto ha establecido una analogía sig. nificativa entre REL1 y DIANA, pero no la especifica de momento].

11:54 E: ¿El rojo [REL1, con misma pregunta que el problema diana] es el que ves más parecido? ¿Exactamente por qué? [El entrevistador demanda especificar el tipo de analogía establecido]

11:56 S: [Piensa en silencio unos segundos]

12:05 DA S: Sí, porque aunque en uno hay globos, y en el otro depósitos de ácido [explicita diferencias superficiales, basadas en los objetos mencionados en los enunciados, entre REL1 y DIANA], en los dos te piden lo mismo [Focaliza la atención en la demanda o incógnita de los problemas]. El problema que he de resolver me pide los 'gramos' y éste también [señala el REL1, que tiene delante]; además tienen las mismas unidades en todo [Explicita una analogía significativa entre REL1 y DIANA basada en el nombre de la magnitud incógnita (similitud superficial). No menciona otras diferencias en objetos y situaciones. No hace mención de rasgos estructurales].

12:20 E: Entonces tú dices que aunque aquí [señala el problema DIANA] hay globos y aquí depósitos [señala el fuente REL1], ambos tienen las mismas unidades y te preguntan por lo mismo [El entrevistador solicita aclaración sobre la irrelevancia de los objetos y situación, y sobre la relevancia de la magnitud incógnita] y tú crees que, de los tres problemas de las fichas de color, ése [señala el REL1] es el que más te va a ayudar...[demanda de revisión de la analogía entre DIANA y REL1, y de la relación posible con los otros fuente].

12:32 UA S: ¡Si, eso! [Utilidad de la analogía. Confirma la relevancia de la incógnita para la analogía establecida entre DIANA y REL1. Persiste la ausencia de mención de rasgos estructurales].

12:49 E: Entiendo lo que me dices... (...) iLo puedes repetir por favor?

12:53 DD S: [Tras breve pausa] Si... Porque estos dos [señala consecutivamente el problema verde SIM1 cuya magnitud incógnita es 'Kilocalorías', y al problema azul ISO1 cuya incógnita son 'microCulombios'] tienen unidades diferentes a éste [señala el DIANA], y te piden cosas diferentes [Explicita diferencias entre DIANA y SIM1, y entre DIANA e ISO1, basadas en las distintas magnitudes incógnita que contienen. no explicita ninguna analogía significativa entre DIANA y SIM1, ni entre DIANA e ISO1].

13:04 E:¿Puedes explicarme eso mejor? ¿A qué te refieres cuando dices 'unidades? [El entrevistador vuelve a solicitar confirmación del rasgo o característica de los problemas sobre las que el sujeto establece las diferencias y las analogías entre ellos]

13:06-13:10 DD S: Sí; estos dos problemas por ejemplo..., en el problema a resolver [DIANA] pone ' $\mathrm{cm}^{3}$ por gramos' y aquí [señala el ISO1] pide 'microCulombios' y 'Voltios'... Y aquí [ahora se refiere al problema SIM1] pide 'Kilocalorías'... ... [Insiste una vez más en tomar como característica clave para comparar problemas la magnitud asociada con la incógnita (nótese que el sujeto dice que el problema "pide...") que es un rasgo superficial]. 
13:25-18:01 El entrevistador cambia las fichas con los enunciados de los fuente, por otras con los mismos problemas pero resueltos. Luego plantea la misma tarea al estudiante y le dice que puede, o no, cambiar de opinión. Con determinación, el sujeto toma primero para leer en silencio y por orden los problemas REL1, SIM1 e ISO1. Al acabar, vuelve a tomar el problema REL1 y se detiene en él. Los tiempos, mayores que los empleados en la fase 2 para leer los problemas, indican que está reprocesándolos, salvando dificultades, y no únicamente comprobando algo predicho. Compara REL1 y DIANA. Luego lee el problema SIM1 muy rápidamente sin traerlo ante sí. Deja el problema rojo REL1 a un lado y toma de nuevo el problema ISO1, lo pone justo debajo del problema DIANA ante sí, y comienza a compararlos con detenimiento.

18:02 E: ¿Qué piensas? ¿Por qué has cambiado de problema de repente?

18:04 GA S: Creo que es éste [la alumna se refiere al problema ISO1,] el que se resuelve igual que éste [el DIANA].

18:06 E: ¿Y porque has cambiado de opinión?

18:10 DA S: Aquí es diferente [la estudiante señala el problema REL1] del problema Diana, porque dice la palabra 'contiene' [En efecto, esta palabra y la frase en la que está inserta, supone una idea de relevancia estructural en el problema fuente REL1. Nótese que antes de ver las ecuaciones explicitadas, el sujeto no atendió a esta idea]. En cambio aquí [problema ISO1. El sujeto compara los fuentes REL1 e ISO1 entre sí por primera vez] dice que 'el condensador va disminuyendo a razón de ...' [Otra vez esta frase tiene relevancia estructural en el problema fuente ISO1. Antes de ver las ecuaciones explicitadas, el sujeto tampoco atendió a esta idea], al igual que en el problema Diana [El sujeto ha atendido también una idea de relevancia estructural del problema DIANA que antes no atendió. Es interesante darse cuenta de que el sujeto, tras estudiar las ecuaciones de los 4 problemas, ha regresado a los enunciados para tratar de comprender cómo se vinculan con las ecuaciones, completando así correctamente la traducción inversa Ecuaciones à Enunciado]. Miro los dos y veo que podría sustituir aquí [en el problema DIANA] estas fórmulas [las ecuaciones del problema ISO1. El sujeto construye una nueva analogía estructural por transformación, para completar la transferencia] porque aunque tengan distintas unidades yo creo que se resolverá igual. [El sujeto declara ahora que la diferencia entre DIANA e ISO1 debida a las diferentes magnitudes mencionadas, no es relevante. Con ello cambia el foco de atención de las fases anteriores, para las analogías y diferencias relevantes entre problemas. Declara que si esos elementos irrelevantes se transforman en los otros, se obtienen problemas equivalentes, es decir, con ecuaciones iguales... lo cual es correcto en el caso del fuente-isomorfo y el Diana].

18:35 E: ¿Y éste [señala el problema SIM1], no crees que te pueda ayudar? [El entrevistador trata de incrementar la fiabilidad de la respuesta, completando las diferencias y similitudes entre problemas].

18:37 DD S: No, porque no es igual que el Diana. En éste [señala el SIM1], los dos 'aumentan a razón de...' 'SSubraya la idea de relevancia estructural del problema similar. Explicita las diferencias entre DIANA y SIM1 en las ideas de los respectivos enunciados que tienen relevancia estructural].

18:44 E: ¿Puedes repetirlo en voz alta?

18:49 DD S: Si. Descarto los problemas rojo [REL1] y verde [SIM1], porque aunque el rojo tenga las DA mismas unidades, al sustituir en el problema diana [se refiere a usar las ecuaciones del probleUA ma REL1 en el DIANA] no lo podría hacer porque aquí tengo cosas diferentes [la estudiante señala la idea de relevancia estructural del enunciado del DIANA, rechazando la importancia de la similitud basada en las magnitudes]. En cambio con éste [señala el problema, ISO1], si sustitu- 
yo según lo que va diciendo el enunciado, isí lo podría poner igual! [Se refiere a que sí podría obtener las mismas ecuaciones. El sujeto comprende que las ideas de relevancia estructural de ambos enunciados son las mismas, y por tanto, el resto de ideas diferentes, pueden ser intercambiadas entre problemas sin alterar sus ecuaciones. Por tanto, declara irrelevantes las diferencias superficiales entre DIANA e ISO1. Ahora, el sujeto recorre el camino resolutivo en el sentido Enunciado à Ecuaciones, cuando en las fases 1 y 2 no lo pudo hacer. La transición a la que pudo acceder, Ecuaciones à Enunciado, le permite ahora realizar también la opuesta completando su comprensión de los problemas.].

19:12 E: Muy bien. ¡Muchísimas gracias por tu colaboración! [Finaliza la entrevista]

\section{Cuadro II. Caso SuperMario64: Análisis del protocolo de la entrevista mos- trando el 'efecto Sísifo'}

Caso SuperMario64: 'Efecto Sísifo', o de retorno del sujeto al uso de criterios superficiales inapropiados, tras haber avanzado en la elaboración de criterios estructurales.

\section{Min y Código Caso: SuperMario64}

Fase 1 (resumen): En la tarea de agrupación, el sujeto definió los conjuntos de problemas según un criterio mixto, que cruzaba la temática con la estructura de los problemas. Durante la fase 1 de la entrevista el sujeto modifica rápidamente su criterio y adopta el de la estructura, que es el de los expertos.

El entrevistador sigue el protocolo: explica la intención de la entrevista y entrega la tarea de agrupación realizada por el sujeto.

$03.20 \quad$ E: ¿Me puedes explicar por qué crees que se resuelven igual, unos sí y otros no?

03:23 S: Mira, entonces lo puse así pero ahora cambiaría y pondría el 1 y el 6 con el 8 y el 3, y el 2 y el 5 con el 4 y el 7 [designa los problemas por su número en el listado suministrado]. [El sujeto al releer su agrupación cambia de criterio y escoge el criterio puramente estructural].

03:30 E: ¿Por qué?

03:31 S: Porque 1, 3, 6 y 8 en todos A aumenta, y B disminuye, y en 2, 5,4 y 7, A aumenta y B aumenta. [El sujeto, durante esta fase 1, ha cambiado su criterio de codificación y categorización de problemas, pasando de uno mixto, en el que la temática tenía importancia para él, a uno que parece puramente estructural: ahora focaliza la atención en una idea de relevancia estructural de los enunciados, proponiendo dos conjuntos: los problemas con estructura Encontrar y aquellos con estructura Alcanzar. No menciona otras ideas]

Fase 2A: analogías y diferencias entre enunciados de problemas

03:35 El entrevistador sigue el mismo protocolo que en el caso anterior.

04:09 GD S: [El Sujeto toma el problema verde SIM1 y lo acerca al problema diana. Lo lee en silencio] Este no me serviría para resolver el Diana.

04:31 E: ¿Por qué crees eso?

04:32 DD S: Porque en el problema a resolver, A disminuye y B aumenta, y aquí [señala el SIM1], A aumenta y B también aumenta. [El sujeto mantiene el criterio que ha adoptado en la fase 1. Percibe una diferencia entre ambos problemas en una idea que tiene relevancia estructural. Esta 
diferencia le parece importante para descartar que ambos se resuelvan igual. No menciona otras similitudes o diferencias entre ellos].

(...)

04:49 GA S: [El sujeto aparta el problema SIM1, toma el problema ISO1 y lo acerca al DIANA]. Este sí.

04:57 E: ¿Ese si te ayudaría? ¿Por qué?

04:59 DA S: Si, porque A disminuye, igual que en problema a resolver, y $\mathbf{B}$ aumenta. [El sujeto insiste en focalizar su atención en esta idea que tiene relevancia estructural, pero solamente en ella. No menciona ninguna otra idea. Al no desarrollar esta similitud, no se puede saber todavía qué tipo de analogía está elaborando].

05:04 E: Déjame que aclare una cosa. Este problema [señala el problema DIANA] te pregunta por los gramos de masa, en cambio este problema [señala el problema ISO1] te está preguntando por los microCulombios, y aun así tú me dices que este te ayudaría a resolver el problema fuente, incluso cuando te están pidiendo cosas distintas.

05:15 S: Si.

05:17 E: ¿Por qué?

05:18 DA S: Porque da igual la unidad con la que lo hagas, si A disminuye, disminuye en ambos y si B aumenta y aumenta en ambos. [El sujeto mantiene el criterio correcto, rechazando la relevancia de la magnitud incógnita. Parece que el sujeto sigue elaborando analogías y diferencias apropiadas...]

05:40-06:10 GA S: [El sujeto toma el problema rojo REL1 y lo acerca para compararlo con el Diana. Lee en silencio]. El problema rojo [REL1] también me serviría de ayuda, porque en éste, aunque no lo diga directamente en el problema, A disminuye y B aumenta [Inferencia correcta, pero aquí se revela un problema importante: pese a focalizar sobre una idea relevante de los enunciados, y rechazar la importancia de elementos superficiales, en realidad el sujeto no ha percibido la estructura de los problemas. Por esa razón, el sujeto no distingue la estructura "mezcla" del problema REL1 de la estructura "encontrar" del problema DIANA].

06:22 E: Entonces, ¿dices que como aquí [señala el REL1] A aumenta y B disminuye también se resuelve igual que el problema diana?

06:26 UA S: Si.

06:28 E:¿Si comparas estos dos [El entrevistador hace referencia a los problemas ISO1 y REL1] ¿Crees que los dos te ayudarían igual?; ¿Ves alguna diferencia entre ellos?

06:46 E: ¿Qué quieres decir? ¿Y esto quiere decir algo? ¿Cuál te ayudaría más a resolver el problema diana?

06:47-7:02 S: [Vuelve a leer pensativamente unos segundos, pero no es capaz de llegar a una conclusión y contestar. Entonces el investigador decide aportar dos problemas fuente más, ISO2 y REL2, por si ayudan a clarificar los procesos mentales del sujeto].

Fase 2B: analogías y diferencias entre enunciados de problemas (continuación)

07:03 El entrevistador entrega 2 problemas-fuente adicionales con cartulinas morada, ISO2 y amarilla, REL2. Ahora el sujeto dispone de 4 problemas fuente, 2 (ISO1 e ISO2) con estructura "encontrar" como el DIANA, y 2 con estructura 'mezcla' (REL1 y REL2).

07:46 GA/DA S: [Lee en silencio los nuevos problemas diana]. Este [ISO2] también serviría porque pasa lo mismo, A disminuye y B aumenta.

07:51 GA S: [El sujeto, acerca el problema REL2 para estudiarlo mejor]. Este también podría ser de ayuda, DA pero ahora ya no lo tengo tan claro porque aquí te dan el dato de la densidad lel sujeto se UA refiere al problema REL2]... No sé seguro si serviría..., pero en teoría, si mantengo la misma 
regla como A disminuye y B aumenta, sí que serviría. [El sujeto, aunque explicita una duda asociada con el concepto de densidad, acaba por atender solo a la idea ya antes señalada, pero obvia el resto de ideas. Esto le lleva al error. No es capaz de completar la traducción y acceder a la estructura de los problemas. A pesar de la relevancia de la idea focalizada, la analogía que establece entre los problemas DIANA, ISO1, ISO2, REL1 y REL2 es por asociación (Clement, 1988), ya que ignora otras ideas importantes que deberían participar también en un 'mapping' adecuado. Sin esas otras ideas no se pueden detectar las diferencias estructurales entre DIANA, ISO1 e ISO2 por un lado, y REL1 y REL2 por el otro]

09:00 E: Entonces dices que aquí [señala el REL2] aparece la 'densidad' y eso te genera dudas... 09:02-09:27 DA S: Sí. Yo estos cuatro [se refiere a ISO1, REL1, ISO2 y REL2], a excepción del dato de la densidad [focaliza ahora en un concepto que también tiene relevancia estructural, y que podría diferenciar entre las estructuras ISO y REL2,] pienso que son bastante parecidos [El sujeto no logra extraer el significado estructural asociado con la presencia de la densidad en REL2, o de la concentración en REL1, en contraste con la idea del aumento o disminución de una cantidad a una razón constante en el DIANA, ISO1 e ISO2. Sigue sin acabar de distinguir entre las estructuras 'mezcla' y 'encontrar'. El investigador decide entonces pasar a la siguiente fase y proporcionar al sujeto los problemas fuente con sus ecuaciones explicitadas, con la esperanza de que al fin consiga percibir las diferencias entre estas dos estructuras].

Fase 3 : analogías y diferencias con los problemas resueltos

El entrevistador sigue el protocolo. Retira todos los problemas fuente y suministra los mismos problemas pero con las ecuaciones explicitadas. Los ubica en las mismas posiciones.

10:30 GA S: [Estudia en silencio. El sujeto compara con mayor interés los problemas ISO1 y REL2]. Los GD resultados son iguales en el azul [ISO1] y el morado [ISO2], pero diferentes con el rojo [REL1] y el amarillo [REL2]. [El sujeto se percata de diferencias en las ecuaciones que resuelven los problemas].

$10: 37$

$10: 46$

11:11 DA E: ¿Pero tú pensabas que esos cuatro se iban a resolver todos con las mismas ecuaciones? S: [El sujeto lee de nuevo y compara los 5 problemas fuente con el problema Diana].

DD

$11: 24$

11:26 UA

S: Creo que es por los datos, porque en éste [señala el problema REL1] pone 'gramos por centímetro cúbico' al igual que éste [señala el REL2] y como en el problema Diana [ijestá diciendo que la causa de que las ecuaciones sean diferentes son las magnitudes implicadas!!]. En cambio aquí [señala el ISO1] pide 'microCulombios por Voltio'. [De forma inesperada, el sujeto cambia el foco de su atención y se centra en las magnitudes asociadas con las temáticas, y en particular, en la magnitud-incógnita].

E: ¿Entonces cuales crees que te ayudarían más a resolver el problema diana?

S: Pues los que tienen $\mathbf{g} / \mathbf{c m}^{3}$ [El sujeto confirma el nuevo criterio erróneo]

E: Tú antes te fijabas sobre todo en que en el problema disminuyera A y aumentara B. En cambio, después de verlos resueltos dices que lo que importa es si el problema trata de gramos o de microCulombios (...). [El entrevistador trata de reconducir al sujeto a la concepción anterior, que era apropiada, y alejarlo de esta nueva concepción equivocada].

11:45 DA S: Pues sí, primero pensaba eso. Creía que la unidad no variaba el resultado pero ahora DD que veo los problemas resueltos y los distintos resultados, los que mantienen la unidad mantienen un resultado igual y los que mantienen unidades diferentes son de diferente resultado [Claramente equivocado, probablemente debido a un mapping erróneo. No entiende el significado de las ecuaciones]

12:12 E: Muy bien, y si ahora tuvieras que escoger que problemas te ayudarían más para resolver el problema diana, ¿cuáles escogerías? 
12:16 UA S: El problema amarillo [REL2] y el rojo [REL1]. [El sujeto persiste en el criterio erróneo. Esto supone un retroceso en el camino para el establecimiento de analogías correctas, tras su avance en las fases 1 y 2. Parece que, en efecto, la analogía de la fase 2, basada en la idea clave aumento / disminución, fue solamente una asociación entre elementos explícitos de los enunciados, pero nunca accedió a la estructura de los problemas. Al no acabar su proceso de transición Enunciadoà Ecuaciones, ni tampoco lograr la transición inversa Ecuaciones à Enunciado, los avances logrados con esfuerzo acaban por desvanecerse y el sujeto regresa a su estado cognitivo original]

$12: 20$

E: Muy bien SuperMario, hemos terminado. Muchísimas gracias por tu colaboración. [Finaliza la entrevista]

\section{Discusión de los casos}

El caso MLR es un ejemplo claro, prototípico, de "efecto pantalla" según el cual las características estructurales de los problemas son ocultadas al sujeto por las superficiales. En el caso anterior, el sujeto no pudo establecer las analogías apropiadas entre problemas porque los rasgos superficiales y explícitos (en este caso, el nombre de las magnitudes-incógnita de los problemas) captaron toda su atención y recibieron una importancia espuria, inadecuada (ver transcripción entre los minutos 11:52 y 13:10, por ejemplo). Sin embargo, una vez es situado por el entrevistador en la representación abstracta, matemática de los problemas, es capaz de comprender el significado de las ecuaciones, y es capaz de asociar las ecuaciones a las ideas clave de los enunciados, completando la "traducción inversa": puede hacer la transición Ecuaciones à Enunciados, pero no la transición Enunciados à Ecuaciones a causa del "apantallamiento". Cuando se retira la "pantalla", el sujeto percibe correctamente lo que se le muestra y entiende todo el proceso de transferencia (ver entrevista a partir del minuto 18:10).

El efecto pantalla es específico de la situación mostrada. Cuando un estudiante no puede realizar la traducción algebraica de los problemas pero tampoco es capaz de entender las ecuaciones en sí mismas, ni su relación con ideas expresadas en los enunciados, no existe "efecto pantalla", ya que este estudiante no puede percibir lo que se le muestra tras las "pantalla", cuando ésta se retire.

Varios son los aspectos particulares que pueden distinguirse en el proceso seguido por el segundo caso, SuperMario64. Como se ha visto en la evolución de los razonamientos de SuperMario64 durante las tres fases de la entrevista, el sujeto establece analogías y diferencias entre problemas, usando un criterio aparentemente adecuado. Sin embargo ese criterio no acaba de ser bien comprendido, al menos no en sus implicaciones, por lo que el sujeto no alcanza el éxito pretendido en la entrevista. La explicación de esto es que las ideas-clave sobre las que razona, que son relevantes para comprender la estructura de los problemas, están en 
realidad explicitadas en los enunciados, (ver entrevista entre los minutos 04:32 y 5:03), por lo que su mención no es garantía de que el sujeto haya llegado a comprender qué significado matemático tienen (ver transcripción a partir del minuto 11:11). En un cierto momento de la entrevista, el sujeto comienza a manifestar su falta de comprensión de la estructura cuando se enfrenta a los problemas 'Relacionados lejano' y 'cercano', cuya estructura es "Mezclas", y afirma que se resuelven igual que el Diana y los 'Isomorfos lejano y cercano', cuya estructura es "Encontrar" (ver 06:10-07:02 y 07:51-09:27). Cuando el investigador presente intenta averiguar si la argumentación sobre ideas-clave de los enunciados conlleva el abandono de criterios superficiales, el sujeto comienza a dudar y aparece el conflicto cognitivo (ver fragmento en el intervalo 05:04-07:02). Este conflicto cognitivo no se resuelve de forma adecuada en la fase 3 de la entrevista, cuando se suministran los cinco problemas fuente correctamente solucionados. Las ecuaciones se convierten para el estudiante en obstáculos de comprensión: no logra entenderlas ni vincularlas con las ideas correspondientes de los enunciados, ni siquiera con las ideas-clave que él mismo tomó como sostén de su criterio. No consigue, por tanto, realizar ninguna de las dos transiciones necesarias, ni la traducción Enunciados à Ecuaciones, ni tampoco la inversa Ecuaciones à Enunciados. Al no lograr alcanzar un estado final estable de comprensión, y hallarse en una situación de conflicto cognitivo, regresa a un estado no-conflictivo para él, sostenido por un criterio incorrecto. Esto supone un retroceso en su avance hacia el aprendizaje. Como Sísifo, cuando empujaba la roca montaña arriba y ésta caía de nuevo antes de alcanzar la cumbre, si el alumno no logra llegar hasta el equilibrio que significa la comprensión, estará condenado a realizar esfuerzos ímprobos continuamente.

\section{Conclusiones y reflexiones didácticas}

El efecto de apantallamiento de lo estructural por lo superficial ha sido encontrado antes en estudios similares de resolución de problemas (GómezFerragud et al, 2013b), y antes, sugerido por Reeves y Weisberg (1994) quienes mostraron la dificultad psicológica de atender lo abstracto, no evidente, pasando más allá de lo superficial y evidente. Estas ideas junto con los resultados empíricos anteriores de naturaleza estadística, parecen apoyar la conjetura de que muchos alumnos de secundaria elaboran modelos mentales analógicos, concretos, del mundo ordinario, antes de intentar elaborar representaciones abstractas, matemáticas de los problemas. En ocasiones, la representación abstracta contiene elementos llamativos que obstaculizan al sujeto construir la representación abstracta adecuada, pues son irrelevantes. El trabajo didáctico consistiría entonces en ayudar a los estudiantes a filtrar y eliminar los elementos irrelevantes, superficiales de los enunciados, para 
aprender a concentrarse solamente en los estructurales. Esto no parece tarea sencilla, e incluso algunos investigadores han sugerido que el éxito en este cometido depende más bien de un factor psicológico interno de los sujetos: la dependencia/independencia de campo (Witkin, Moore, Godenough y Cox, 1975). Los estudiantes independientes de campo son capaces de abstraer con facilidad los elementos importantes, comunes a varias situaciones particulares, y alcanzar la estructura de las mismas rápidamente, mientras los dependientes de campo dependen fuertemente de los contextos particulares para comprender los conceptos implicados, por lo que tienen dificultades en generalizar y abstraer. En este caso, la pregunta sería, ¿pueden los profesores ayudar de algún modo a los estudiantes dependientes a ser independientes de campo? En todo caso, los profesores deben ser conscientes de la dificultad grande que el proceso de traducción algebraica implica. Cuando enseñan a sus estudiantes a resolver problemas, y resuelven varios ejemplos apropiados, en realidad están ayudando a esos estudiantes a conectar la resolución matemática con el enunciado (Ecuaciones à Enunciado). La comprensión de este proceso de "traducción algebraica inversa" no implica la comprensión del proceso deseado, Enunciado à Ecuaciones. Hay que tratar de evitar la confusión entre los dos sentidos de la transición para lograr el éxito instruccional.

El segundo caso nos permite conocer, no sólo un efecto indeseado en el proceso de comprensión, sino también un fenómeno didáctico de importancia para los profesores. Este fenómeno didáctico está motivado por la 'apariencia de comprensión' que el sujeto muestra cuando sostiene que las analogías y diferencias entre problemas estriban en la igualdad o diferencia en ciertas ideas clave (ver Tabla II). El 'efecto Sísifo' mostrado en la entrevista -o de 'retroceso' del sujeto tras un avance incompleto-, se produce como consecuencia de la imposibilidad del sujeto de alcanzar un nuevo estado cognitivo de estabilidad, asociado con una comprensión completa de las analogías y diferencias en la estructura algebraica de los problemas. No completar el proceso de elaboración de una representación mental adecuada de todos esos problemas, acaba por suponer un fracaso didáctico. Los profesores deberían prestar atención a la diferencia entre una 'apariencia de comprensión' y la comprensión real. Cuando el sujeto expresa una idea de relevancia estructural para sostener sus argumentos, se puede producir en los profesores una ilusión de que el sujeto está comprendiendo cuando, en realidad, no es así. Esto puede conducir a relajar el trabajo instruccional necesario para llevar al alumno definitivamente a la representación abstracta de los problemas. Esta ilusión está provocada por las inferencias que el propio profesor elabora para conectar la parte de la información (las ideas clave) mencionadas por el alumno, que en realidad están explícitas en los enunciados, con otra parte (las estructuras de los problemas) que están 
implícitas. Esto recuerda uno de los fenómenos didácticos que Brousseau definió como "efecto Jourdain" (Brousseau, 1986) según el cual, el profesor intelectualiza y da importancia a ciertas respuestas y comportamientos de los alumnos en los que reconoce indicios de conocimiento, cuando en realidad estas respuestas y comportamientos tienen causas, motivaciones y significados triviales. Este caso nos previene de trivializar los procesos mentales de los sujetos cuando tratan de establecer analogías estructurales entre problemas, y nos hace ver que los estudiantes se encuentran grandes obstáculos en el camino de la comprensión de la transferencia analógica, que es una de las estrategias más usadas en las aulas para enseñar a resolver problemas.

\section{Referencias Bibliográficas}

ARTZT, A. y ARMOUR-THOMAS, E. (1992). Development of a cognitivemetacognitive framework for protocol analysis of mathematical problem solving in small groups. Cognition and Instruction, 9 (2), pp. 137-175. doi: 10.1207/s1532690xci0902_3

BERNARDO, A.B.I. (2001). Analogical problem construction and transfer in mathematical problem solving. Educational Psychology, 21(2), pp. 137-150.

BROUSSEAU, G. (1986). Fondaments et méthodes de la didactique des Mathématiques. Recherches en Didactique des Mathématiques, 7(2), pp. 33-115.
CASTRO, E.; RICO, L.; BATANERO, C. y CASTRO, E. (1991). Dificultad en problemas de comparación multiplicativa. En F. Furinghetti (Ed) Proceedings Fifteenth PME Conference Vol 1. Assisi, Italy, (pp 192- 198).

CHEN, Z. y KLAHR, D. (2008). Bridging the gap: Remote transfer of problemsolving and scientific reasoning strategies in children. In R. Kail (Ed.), Advances in child development and behavior. Vol. 36, Burlington, MA: Academic Press, (pp. 419-470)

CHI, M.T.H., FELTOVICH, P.J. y GLASER, R. (1981). Categorization and representation of physics problems by experts and novices. Cognitive Science, 5, pp. 121-152.

CLEMENT, J.(1988). Observed methods to generate analogies in scientific problem solving. Cognitive Science, 12 , pp. 563-586.

CODINA, A.; CASTRO, E. y CAÑADAS, M.C. (2011). Un sistema de categorías para el análisis de la interactividad en una i-actividad de resolución de problemas. En J.L. Lupiañez, M. C. Cañadas, M. Molina, M. M. Palarea y A. Maz (Eds), Investigaciones en pensamiento numérico y algebraico e historia de la matemática y educación matemática. Granada: Grupo de pensamiento numérico y algebraico e historia de la matemática y educación matemática, (pp. 157-164)

GENTNER, D. (1983). Structuremapping. A theoretical framework for analogy. Cognitive Science, 7, 
pp. 155-170. doi: 10.1016/S03640213(83)80009-3.

GICK, M.L. y HOLYOAK, K.J. (1983). Schema induction and analogical transfer. Cognitive Psychology, 15, pp. 1-38.

GÓMEZ-FERRAGUD, C.B.; SOLAZPORTOLÉS, J.J. y SANJOSÉ, V. (2012). Una revisión de los procesos de transferencia para el aprendizaje y enseñanza de las ciencias. Didáctica de las Ciencias Experimentales y Sociales, vol. 26, pp. 199-227.

GÓMEZ-FERRAGUD, C.B., SOLAZPORTOLÉS, J.J. y SANJOSÉ, V. (2013a). Efectos de la similitud superficial y estructural sobre la transferencia a partir de análogos en problemas de alta y baja familiaridad: primeros resultados. Enseñanza de las Ciencias, 31(1), pp.135-151. GÓMEZ-FERRAGUD, C.B.; SOLAZPORTOLÉS, J.J. y SANJOSÉ, V. (2013b). Analogy Construction and Success in Mathematics and Science Problem-Solving: a Study with Secondary Students. Revista de Psicodidáctica, 18(1), pp. 81-108.

GÓMEZ-FERRAGUD, C.B., SOLAZPORTOLÉS, J.J. y SANJOSÉ, V. (2013c). Dificultades para codificar, relacionar y categorizar problemas verbales algebraicos: Dos estudios con alumnos de secundaría y profesores en formación. Pendiente de revisión en Bolema.

HOLYOAK, K.J. (1984). Analogical thinking and human intelligence. In
R.J. Sternberg (Ed). Advances in the psychology of human intelligence, vol. 2. Hilsdale, NJ: Erlbaum, (pp. 199-230).

HOLYOAK, K.J., y KOH, K. (1987). Surface and structural similarity in analogical transfer. Memory and Cognition, 15(4), pp. 332-340. doi: 10.3758/BF3197035

NOVICK, L. (1988). Analogical transfer, problem similarity, and expertise. Journal of Experimental Psychology: Learning, Memory and Cognition, 14, pp. 510-520. Disponible en: http://psycnet.apa.org/index. $\mathrm{cfm}$ ?fa = search.displayRecord $\& \mathrm{u}$ id=1988-31644-001

REED, S.K., DEMPSTER, A. y ETTINGER, M. (1985). Usefulness of analogous solutions for solving algebra word problems. Journal of Experimental Psychology:Learning, Memory and Cognition, 11, pp. 106-125.

REED, S.K. (1987). A structuremapping model for word problems. Journal of Experimental Psychology: Learning, Memory and Cognition, 13, pp. 124- 139.

SHOENFELD, A. (1985). Mathematical problem solving. Orlando: Academic Press.

WITKIN, H.A., MOORE, C.A., GODENOUGH, D.R. y COX, P.W. (1975). Field-dependent and fieldindependent cognitive styles and their educational implications. Research bulletin. Princeton, New Jersey: Educational Testing Service 


\section{ANEXO 1: PROBLEMAS UTILIZADOS EN LA TAREA DE AGRUPACIÓN}

Los 8 problemas de esta tarea se obtuvieron de un diseño 2X2X2 (estructura X temática $\mathrm{X}$ magnitud incógnita), manteniendo común el máximo de elementos sintácticos. (Entre paréntesis los cambios de uno a otro problema en el enunciado y en la pregunta).

\section{Estructura Encontrar}

Enunciados: Consideremos dos globos A y B de diferente tamaño y fabricados con distinto material. Inicialmente el globo A tiene un volumen de $2000 \mathrm{~cm}^{3} \mathrm{y}$ el globo B está vacío. Entonces se conectan a la vez dos bombas térmicas (neumáticas) idénticas, una que toma calor (extrae gas) de A y lo transfiere a (introduce en) un acumulador (depósito) y otra que toma el calor (extrae el gas) del acumulador (depósito) y lo transfiere a (introduce en) B. El globo A va disminuyendo su volumen a razón de $20 \mathrm{~cm}^{3} / \mathrm{cal}$ $\left(\mathrm{cm}^{3} / \mathrm{g}\right)$ y el globo B va aumentando su volumen a razón de $30 \mathrm{~cm}^{3} / \mathrm{cal}\left(\mathrm{cm}^{3} / \mathrm{g}\right)$.

Preguntas: ¿Cuántas calorías (gramos) se habrán transferido de A a $\mathrm{B}$, cuando sus volúmenes sean iguales? (¿Qué volumen habrá en A y en B cuando sus volúmenes sean iguales?)

\section{Estructura Alcanzar}

Enunciados: Consideremos dos globos A y B de diferente tamaño y fabricados con distinto material. Inicialmente el globo A tiene un volumen de $2000 \mathrm{~cm}^{3}$ y el globo B está vacío. Entonces se conectan a la vez dos bombas térmicas (neumáticas) idénticas, que extraen calor (toman gas) de un acumulador (depósito) y lo transfieren a (introducen en) cada globo. El globo A va aumentando su volumen a razón de $20 \mathrm{~cm}^{3} / \mathrm{cal}$ $\left(\mathrm{cm}^{3} / \mathrm{g}\right)$ y el globo B va aumentando su volumen a razón de $30 \mathrm{~cm}^{3} / \mathrm{cal}\left(\mathrm{cm}^{3} / \mathrm{g}\right)$.

Preguntas: ¿Cuántas calorías (gramos) se habrán transferido a A y a B, cuando sus volúmenes sean iguales? (¿Qué volumen habrá en A y en B cuando sus volúmenes sean iguales?)

Nota: Para reducir la extensión ocupada, entre paréntesis se indican las diferencias entre enunciados de los 8 problemas utilizados en la prueba. Téngase en cuenta que se ha indicado que los globos son de distinto material para permitir que el gas en su interior esté sometido a distintas presiones. 


\section{ANEXO 2: PROBLEMAS UTILIZADOS EN LA PRUEBA DE TRANSFER (FASE 2 DE LAS ENTREVISTAS)}

\section{PROBLEMA DIANA}

Consideremos dos globos A y B de diferente tamaño y fabricados con distinto material. Inicialmente el globo A tiene un volumen de $2000 \mathrm{~cm}^{3}$ y el globo B está vacío. Entonces se conectan a la vez dos bombas neumáticas idénticas, una que extrae gas de A y lo introduce en un depósito y otra que extrae el gas del depósito y lo introduce en B. El globo A va disminuyendo su volumen a razón de $20 \mathrm{~cm}^{3} / \mathrm{g}$ y el globo $B$ va aumentando su volumen a razón de $30 \mathrm{~cm}^{3} / \mathrm{g}$.

¿Cuántos gramos de gas se habrán transferido de $\mathrm{A}$ a $\mathrm{B}$, cuando sus volúmenes sean iguales?

\section{PROBLEMAS FUENTE LEJANOS (FASE 2A)}

(Similar: SIM1) Consideremos dos globos A y B de diferente tamaño y fabricados con distinto material. Inicialmente el globo A tiene un volumen de $2000 \mathrm{~cm}^{3}$ y el globo B está vacío. Entonces se conectan a la vez dos bombas térmicas idénticas que extraen calor de un acumulador y lo transfieren a cada globo. El globo A va aumentando su volumen a razón de $20 \mathrm{~cm}^{3} / \mathrm{kilocaloría} \mathrm{y} \mathrm{el} \mathrm{globo} \mathrm{B} \mathrm{va} \mathrm{aumentan-}$ do su volumen a razón de $30 \mathrm{~cm}^{3} /$ kilocaloría.

¿Cuántas kilocalorías se habrán transferido a A y a B cuando sus volúmenes sean iguales?

(Isomorfo: ISO1) Dos condensadores eléctricos A y B diferentes están conectados a un circuito eléctrico. Inicialmente el condensador A tiene una diferencia de potencial eléctrico entre sus bornes 2000 Voltios mayor que B. Entonces se conectan a la vez dos dispositivos idénticos, uno que extrae carga de A y la transfiere a un acumulador y otro que extrae la carga del acumulador y la transfiere a B. El condensador A va disminuyendo su potencial a razón de $20 \mathrm{Volt} / \mathrm{microculombio} \mathrm{y}$ el condensador $\mathrm{B}$ va aumentando su potencial a razón de $30 \mathrm{Volt} / \mathrm{microculombio.}$

¿Cuántos microculombios se habrán transferido de A a B cuando sus diferencias de potencial sean iguales?

(Relacionado: REL1) Consideremos dos depósitos A y B diferentes, con disolución de ácido ascórbico en agua. Inicialmente el contenedor A contiene un volumen $2000 \mathrm{~cm}^{3}$ de disolución más que B. Entonces se conectan a la vez dos bombas hidráulicas idénticas, una que extrae disolución de A y la introduce en un conte- 
nedor vacío y otra que extrae la disolución del contenedor y la introduce en B. La disolución A contiene ácido con densidad de $20 \mathrm{~g} / \mathrm{cm}^{3}$ y la disolución B contiene ácido con una densidad de $30 \mathrm{~g} / \mathrm{cm}^{3}$.

¿Cuántos gramos de ácido ascórbico se habrán transferido de A a B cuando sus volúmenes sean iguales?

\section{PROBLEMAS FUENTE CERCANOS (FASE 2B)}

(Relacionado: REL2) Consideremos dos globos A y B de diferente tamaño y fabricados con distinto material. Inicialmente el globo A tiene un volumen 2000 $\mathrm{cm}^{3}$ mayor que B. Entonces se conectan a la vez dos bombas neumáticas idénticas, una que extrae gas licuado de A y lo introduce en un depósito vacío y otra que extrae el gas licuado del depósito y lo introduce en $\mathrm{B}$. El globo A tiene un gas licuado con una densidad de $20 \mathrm{~g} / \mathrm{cm}^{3}$ y el globo B tiene un gas licuado con una densidad de $30 \mathrm{~g} / \mathrm{cm}^{3}$.

¿Cuántos gramos de gas licuado se habrán transferido de A a B, cuando sus volúmenes sean iguales?

(Isomorfo: ISO2) Consideremos dos depósitos A y B de diferente tamaño con disolución de ácido ascórbico en agua. Inicialmente el depósito A contiene un volumen $2000 \mathrm{~cm}^{3}$ de disolución y el depósito B está vacío. Entonces se conectan a la vez dos bombas hidráulicas idénticas, una que extrae disolución de A y la introduce en un contenedor con agua, y otra que extrae disolución del contenedor y la introduce en B. La disolución A va disminuyendo su volumen a razón de $20 \mathrm{~cm}^{3} / \mathrm{g}$ de ácido y la disolución $\mathrm{B}$ va aumentando su volumen a razón de $30 \mathrm{~cm} 3 / \mathrm{g}$ de ácido. ¿Cuántos gramos de ácido ascórbico se habrán transferido de $\mathrm{A}$ a $\mathrm{B}$ cuando sus volúmenes sean iguales? 